\title{
Some Results on the Variation of Composite Function of Functions of Bounded $d$ Variation
}

\author{
Mohsen Soltanifar* \\ Department of Mathematics \\ Faculty of Science \\ K.N. Toosi University of Technology \\ P.O. Box 16315-1618 Tehran IRAN
}

\begin{abstract}
In this paper we discuss conditions under which the composite function of two functions of bounded $d$ variation is also of bounded $d$ variation.
\end{abstract}

\section{INTRODUCTION}

Camille Jordan

(1838-1922)

introduced the class of all functions of bounded variation, and classed them as differences of non-decreasing functions. These functions play a central role in many investigations, notably in studies of rectifiability and in studies into fundamental questions involving integrals and derivatives. They also lead to natural generalizations in the abstract studies of measure and integration. Today, many text books in real analysis cover them in relation to the Riemann-Stieltjes integrability of a real valued function $f$ defined on the closed interval $[a, b]$. [1-3] By considering these functions, we see that for the Euclidean metric $d_{E}$, the bounded $d_{E}$ variation is invariant under arithmetic operations + , -, and $\mathrm{x}$; that is, the arithmetic operations,+- , and $x$ with two functions $f$ and $g$ of bounded variation on $[a, b]$ with respect to the Euclidean metric $d_{E}$ give functions $f \pm g$ and $f \times g$ of bounded variation of $[a, b]$ with respect to the same metric. (N.B. The bounded $d_{E}$ variation is not invariant under the arithmetic operation $\div$ because in the

*Contact: soltanifar@sina.kntu.ac.ir division case the denominator function may not be far from zero; that is, $0 \in(\operatorname{Im}|g|)^{-}$ and consequently the function $1 \div g$ is unbounded on $[a, b]$ and so is not a bounded variation on $[a, b]$.) As we have seen before, the results of a composition are always studied and discussed along wit the results of arithmetic operations,,$+- \times$, and $\div$ in subjects like continuity, differentiability and integrability [4]. In this paper, Chistyako's 1997 definition of bounded $d$ variation is used to deal with the possible results of variation of a composite function $g \circ f$ of two functions of bounded $d$ variation $f$ and $g$ defined on $[a, b]$ and $[c, d]$ respectively, with $\operatorname{Im}(f) \subseteq[c, d]$. In particular, for the case $d=d_{E}$ the results and the above mentioned results on arithmetic operations,,$+- \times$, and $\div$ make a consistent and complete discussion of all primary operations of functions in the subject of functions of bounded $d_{E}$ variation versus the consistent and complete discussion of all primary operations of function of continuity, differentiability and integrability of functions.

For the Euclidean metric $d_{E}$ case, it is showed that despite the arithmetic operations,+- , and $x$, and similar to the 
arithmetic operation $\div$, bounded $d_{E}$ variation is not invariant under the composition operation. In the first two theorems, some conditions of tow functions of bounded $d_{E}$ variation are represented that imply their composite function is also of bounded $d_{E}$ variation. By considering a general metric $d$ instead of $d_{E}$, in the third theorem a necessary and non-sufficient condition of the metric $d$ is stated to have the property of the invariance of bounded $d$ variation under the composition operation. The last theorem deals with determining a category of metrics $d$ that have the property of the invariance of bounded $d$ variation under composition.

\section{PRELIMINARIES}

The reader who has studied the concept of functions of bounded variation should be well acquainted with the following definitions and results. For an essential account of the theory of function of bounded variation with respect to a metric $d$, see reference [5]. In the discussion throughout this paper it is assumed that the metric $d$ is considered as a real-valued function from the 2-dimensional Euclidean space $\left(\mathfrak{R}^{2}, d_{E}{ }^{2}\right)$ into the 1-dimensional Euclidean space $\left(\mathfrak{R}, \mathrm{d}_{\mathrm{E}}\right)$.

Furthermore, it is taken for granted that both functions $f$ and $g$ are defined on bounded closed intervals in the real line. For the Main Results some notations and definitions are needed. We denote the set of all Partitions of $[a, b]$ by $\mathcal{P}_{b}^{a}$. That is,

$$
\left.\mathcal{P}_{b}^{a}=P=\left\{a_{k}\right\}_{k=0}^{n} \subseteq[a, b]: n \in \mathrm{N}, a=a_{0}<a_{1}<\ldots<a_{n-1}<a_{n}=b\right\}
$$

Let $f$ be a function defined on $[a, b]$. For each partition $P=\left\{a_{k}\right\}_{k=0}^{n}$ of $[a, b]$, put $V_{d}(f, P)=\sum_{k=1}^{n} d\left(f\left(a_{k}\right), f\left(a_{k-1}\right)\right)$. The number $V_{d}(f, P)$ is called the variation of $f$ on $[a, b]$ for partition $P$.

Definition 1. The function $f$ is said to be of bounded $d$ variation on $[a, b]$ provided the set $\left\{V_{d}(f, P): P \in \mathcal{P}_{b}^{a}\right\}$ is bounded above. If $f$ is a function of bounded $d$ variation then we set $V_{d}(f,[a, b])=\sup \left\{V_{d}(f, P): P \in \mathcal{P}_{b}^{a}\right\}$. We call $V_{d}(f,[a, b])$ the variation of $f$ on $[a, b]$ with respect to metric $d$.

Definition 2. The metric $d$ is said to have the property of the Invariance of Bounded $d$ Variation under Composition Operation (IBVCO property) if, for any two function $f$ and $g$ of bounded $d$ variation on $[a, b]$ and $[c, d]$, respectively, with $\operatorname{Im}(f) \subseteq[c, d], g \circ f$ Is a function of bounded $d$ variation on $[a, b]$.

The following result from proposition 2.4.3 of [6] (pages 39-40) will be useful in the rest of this paper.
Proposition 1. Let $f$ be a function defined on $[a, b]$ and $d_{1}, d_{2}$ are two Lipschitz equivalent metrics. Then, $f$ is a bounded $d_{1}$ variation on $[a, b]$ if and only if it is a bounded $d_{2}$ variation on $[a, b]$.

The following corollary is a straightforward generalization of Theorem 6.3.6 of reference [1] (page 321).

Corollary 1. Suppose that $f$ is a function of bounded $d_{\mathrm{E}}$ variation on $[a, b]$ and $\left\{a_{k}\right\}_{k=0}^{n}$ is a partition of $[a, b]$. Then $f$ is a function of bounded $d_{E}$ variation on each $\left[a_{k-1}, a_{k}\right]$, and furthermore:

$$
\begin{array}{r}
\sum_{k=1}^{n} V_{d_{E}}\left(f,\left[a_{k-1}, a_{k}\right]\right)=V_{d_{E}}(f,[a, b]) \\
V_{d_{E}}\left(f,\left[a_{k-1}, a_{k}\right]\right) \leq V_{d_{E}}(f,[a, b]) \\
\text { for } k=1,2 \ldots \mathrm{n} .
\end{array}
$$

\section{MAIN RESULTS}

In this section we intend to show that the metric $d$ plays an important role in boundedness of total variation of composite functions. At this stage we mention that all theorems below with respect to metric $d$ are 
valid with little changes for all Lipschitz equivalent metrics with d (see Proposition 2).

We begin with the rather concrete case $d=d_{E}$. To have a similar theorem to the theorems of arithmetic operations + , -, and $x$, we naturally expect to give an affirmative answer to the following question.

Question 1. Does the metric $d_{E}$ have the IBVCO property?

On the other hand, the counter examples below give a negative answer to the question.

Example 1. Consider functions of bounded $d_{E}$ variation like $f$ and $g$ defined on $[0,1]$ and $[-1,1]$, respectively by: $f(x)=x^{2} \sin (\pi / x)$ if $(x \neq 0), f(0)=0$ and $g(x)=1$ if $(x \neq 0), g(0)$ $=0$. Then the composite function $g \circ f$ is defined on [0,1] by: $g \circ f(x)=0$ if $(x=0,1 / n$ with $n=1,2,3 \ldots)$ and $g \circ f(x)=1$ if $(x \neq 0$,
$1 / n \quad$ with $n=1,2,3 \ldots)$ Put $P_{n}=\left\{0, \frac{1}{n}, \frac{1}{2}\left(\frac{1}{n}+\frac{1}{n-1}\right), \frac{1}{n-1}, \ldots, \frac{1}{2}, \frac{1}{2}\left(\frac{1}{2}+1\right), 1\right\}$ where $n=2,3 \ldots$ Then $P_{n}$ is a partition of $[0,1]$ and $V_{d_{E}}\left(g \circ f, P_{n}\right)=2(n-1)$ where $n$ $=2,3 \ldots$ Accordingly, $V_{d_{E}}(g \circ f,[0,1])=\infty$.

Example 2. Consider functions of bounded $d_{E}$ variation like $f$ and $g$ defined on $[0,1]$ respectively by: $f(x)=1 / n^{2} \quad$ if $\left(x=\frac{n-1}{n}, n=1,2 \ldots\right)$, and $f(x)=0 \quad$ if $\left(x \neq \frac{n-1}{n}, n=1,2 \ldots\right)$ and $g(x)=x^{\frac{1}{2}}$. Then the composite function $g \circ f$ is defined on $[0,1]$ by: $g \circ f(x)=\frac{1}{n} \quad$ if $\left(x=\frac{n-1}{n}, \quad n=1,2,3 \ldots\right)$ and $g \circ f(x)=0$ if $\left(x \neq \frac{n-1}{n}, \quad n=1,2,3 \ldots\right)$. Put

$$
P_{n}=\left\{0, \frac{1}{2}(0+1), \frac{1}{2}, \frac{1}{2}\left(\frac{1}{2}+\frac{2}{3}\right), \ldots \frac{n-2}{n-1}, \frac{1}{2}\left(\frac{n-2}{n-1}+\frac{n-1}{n}\right), \frac{n-1}{n}, 1\right\}
$$

where $\mathrm{n}=2,3 \ldots$ Then $P_{n}$ is partition of $[0,1]$ and $V_{d_{E}}\left(g \circ f, P_{n}\right)=2 \sum_{k=1}^{n} \frac{1}{k}-1$ where $\mathrm{n}=$ $2,3 \ldots$ Accordingly, $V_{d_{E}}(g \circ f,[0,1])=\infty$.

The counterexamples above show that the composition of two functions of bounded $d_{E}$ variation is not necessarily itself of bounded $d_{E}$ variation. Nevertheless, there exist remarkable and special cases in the composite function of two function of bounded $d_{E}$ variation is of bounded $d_{E}$ variation too. As the first case, we suppose that $g$ is an arbitrary function of bounded $d_{E}$ variation on $[c, d]$. We ask if there are sufficient conditions of the function $f$ of $d_{E}$ variation on $[a, b]$ with $\operatorname{Im}(f) \subseteq[c, d]$ implying the bounded $d_{E}$ variation of the composite function $g \circ f$ on $[a, b]$. By observing a couple of counterexamples we can see that the function $f$ in both counterexamples has a common property, that is, there is not any partition $\left\{a_{k}\right\}_{k=0}^{n}$ of $[a, b]$ which $f$ is monotone on each $\left\lfloor a_{k-1}, a_{k}\right\rfloor$ for $k=1,2 \ldots n$. If we eliminate this common property, then we are led to the following theorem.

Theorem 1. Let $f$ be an arbitrary function of bounded $d_{E}$ variation on $[a, b]$, and let there exist a partition $\left\{a_{k}\right\}_{k=0}^{n}$ of $[a, b]$ which $f$ is monotone on each $\left\lfloor a_{k-1}, a_{k}\right\rfloor$ for $k=$ $1,2 \ldots n$. Then, for any function $g$ of bounded $d_{E}$ variation on $[c, d]$ with $\operatorname{Im}(f) \subseteq[c, d], \quad g \circ f$ is bounded $d_{E}$ variation on $[a, b]$, and furthermore:

$$
V_{d_{E}}(g \circ f,[a, b]) \leq n V_{d_{E}}(g,[c, d]) .
$$

Proof. Let $1 \leq \mathrm{k} \leq n$ and suppose that $f$ is increasing on $\left\lfloor a_{k-1}, a_{k}\right\rfloor$ (the proof is analogous in the other case). Let 
$P=\left\{a_{l}^{*}\right\}_{l=0}^{m}$ be a partition of $\left[a_{k-1}, a_{k}\right\rfloor$, so $P^{*}=\left\{f\left(a_{l}^{*}\right)\right\}_{l=0}^{m}$ is a partition of $\left\lfloor f\left(a_{k-1}\right), f\left(a_{k}\right)\right\rfloor \quad$ (see Equation2 of Corollary 1$)$ and:

$$
V_{d_{E}}\left(g,\left\lfloor f\left(a_{k-1}\right), f\left(a_{k}\right)\right\rfloor\right) \leq V_{d_{E}}(g,[c, d])
$$

We also have:

$$
\begin{aligned}
V_{d_{E}}(g \circ f, P) & =\sum_{l=1}^{m} d_{E}\left(g \circ f\left(a_{l}^{*}\right), g \circ f\left(a_{l-1}^{*}\right)\right) \\
& =\sum_{l=1}^{m} d_{E}\left(g\left(f\left(a_{l}^{*}\right)\right), g\left(f\left(a_{l-1}^{*}\right)\right)\right. \\
& =V_{d_{E}}\left(g, P^{*}\right) .
\end{aligned}
$$

Hence, we can write:

$$
\begin{aligned}
V_{d_{E}}\left(g \circ f,\left[a_{k-1}, a_{k}\right]\right) & =\sup \left\{V_{d_{E}}(g \circ f, P): P \in \mathcal{P}_{a_{k-1}}^{a_{k}}\right\} \\
& \leq \sup \left\{V_{d_{E}}\left(g, P^{*}\right): P^{*} \in \mathcal{P}_{f\left(a_{k-1}\right)}^{f\left(a_{k}\right)}\right\} \\
& =V_{d_{E}}\left(g,\left\lfloor f\left(a_{k-1}\right), f\left(a_{k}\right)\right]\right) .
\end{aligned}
$$

Now equations (4) and (5) give

$$
V_{d_{E}}\left(g \circ f,\left[a_{k-1}, a_{k}\right]\right) \leq V_{d_{E}}(g,[c, d]) \text { for } k=1,2 \ldots n .
$$

Taking summation on these inequalities implies:

$$
\sum_{k=1}^{n} V_{d_{E}}\left(g \circ f,\left[a_{k-1}, a_{k}\right]\right) \leq n V_{d_{E}}(g,[c, d]) .
$$

Therefore by considering equation (1) of Corollary 1 the desired statement is proved.

Corollary 2. Let $f(x)=\sum_{k=1}^{n} a_{k} x^{k}$ be a polynomial with real coefficients defined on $[a, b]$. If $g$ is a function of bounded $d_{E}$ variation on $[c, d]$ such that $\operatorname{Im}(f) \subseteq[c, d]$, then $g \circ f$ is a function of bounded $d_{E}$ variation on $[a, b]$.

Now, as the second case, we consider $g$ instead of $f$. Suppose that $f$ is an arbitrary function of bounded $d_{E}$ variation on $[a, b]$. We ask if there are sufficient conditions of the function $g$ of bounded $d_{E}$ variation on $[c, d]$ with $\operatorname{Im}(f) \subseteq[c, d]$ implying the bounded $d_{E}$ variation of the composite function $g \circ f$ on $[a, b]$. On page 406 of reference [7], it has been proved that every function $g$ of bounded $d_{E}$ variation is almost everywhere differentiable. If $g$ is not differentiable at even one point of $(c, d)$ then Example 1 shows that $g$ is an inappropriate choice for our purpose, so we have to add the limitation of differentiability of $g$ on $(c, d)$. 
Furthermore, if $g$ is differentiable on $(c, d)$ but $g^{\prime}$ is unbounded, then Example 2 shows that $g$ is also an inappropriate choice for our purpose. If we add the boundedness of $g^{\prime}$ on $(c, d)$ as the second limitation, then we find the appropriate choice, and this leads us to the next theorem.
Theorem 2. Let $f$ be an arbitrary function of bounded $d_{E}$ variation on $[a, b]$. Then, for any function $g$ of bounded $d_{E}$ variation on $[c, d]$ with bounded derivative $g^{\prime}$ on $(c, d)$ and $\operatorname{Im}(f) \subseteq[c, d], g \circ f$ is a function of bounded $d_{E}$ variation on $[a, b]$ and furthermore:

$$
V_{d_{E}}(g \circ f,[a, b]) \leq 4\left(\sup _{c \leq x \leq d}|g(x)|\right)+\left(\sup _{c<x<d}\left|g^{\prime}(x)\right|\right) V_{d_{E}}(f,[a, b])
$$

Proof. By means of Corollary 4 of reference

7 (page 142), for every $x, y \in(c, d)$ :

$$
\begin{aligned}
& d_{E}(g(x), g(y)) \\
& \quad \leq\left(\sup _{c<x<d}\left|g^{\prime}(x)\right|\right) d_{E}(x, y)
\end{aligned}
$$

Now the above inequality, and the arbitrarilygiven partition $P=\left\{a_{k}\right\}_{k=0}^{n}$ of $[a, b]$, give:

$$
\begin{aligned}
V_{d_{E}}(g \circ f, P) & =\sum_{k=1}^{n} d_{E}\left(g \circ f\left(a_{k}\right), g \circ f\left(a_{k-1}\right)\right) \\
& =d_{E}\left(g \circ f\left(a_{1}\right), g\left(a_{0}\right)\right)+\sum_{k=2}^{n-1} d_{E}\left(g \circ f\left(a_{k}\right), g \circ f\left(a_{k-1}\right)\right) \\
& \left.=d_{E}\left(g\left(f\left(a_{1}\right)\right), g\left(f\left(a_{0}\right)\right)\right)+\sum_{k=2}^{n-1} d_{E}\left(g \circ f\left(a_{n}\right), g \circ f\left(a_{n-1}\right)\right), g\left(f\left(a_{k-1}\right)\right)\right) \\
& <4\left(\sup _{c<x<d}\left|g^{\prime}(x)\right|\right) V_{d_{E}}(f, P) .
\end{aligned}
$$

Therefore, we can conclude that:

$$
\begin{array}{r}
V_{d_{E}}(g \circ f,[a, b])=\sup \left\{V_{d_{E}}(g \circ f, P): P \in \mathcal{P}_{a}^{b}\right\} \\
\leq 4\left(\sup _{c \leq x \leq d}|g(x)|\right)+\left(\sup _{c<x<d}\left|g^{\prime}(x)\right|\right) V_{d_{E}}(f,[a, b]) .
\end{array}
$$

Corollary 3. Let $f$ be a function of bounded $d_{E}$ variation on $[a, b]$, then $\exp (f)$ is a function of bounded $d_{E}$ variation on the same interval.

Corollary 4. Suppose function $f$, positive and far from zero, and function $g$ are both bounded $d_{E}$ variation on $[a, b]$. Then the function $f^{g}$ is also of bounded $d_{E}$ variation on $[a, b]$.

Proof. By means of Theorem 2, $\ln (f)$ is a function of bounded $d_{E}$ variation and so is $g \ln (f)$. Now Corollary 3 implies the bounded $\mathrm{d}_{\mathrm{E}}$ variation of $\exp (g \ln (f))$ or $f^{g}$. 
In sum, the above theorems and results show that the Euclidean metric $d_{E}$ does not have the IBVCO property. The following theorem gives a characterization of the metric $d$ which has the IBVCO property and also, as a corollary it shows that the metric $d_{E}$ does not have the necessary condition of having that property.

Theorem 3. Let the metric $d$ have the IBVCO property. Then, the metric $d$ as a real valued function

$$
\mathrm{d}:\left(\mathfrak{R}^{2}, d_{E^{2}}\right) \rightarrow\left(\mathfrak{R}, d_{E}\right)
$$

is discontinuous.

Proof. Assume, to reach a contradiction, that the metric $d$ is continuous. Consider the increasing sequence $\left\{y_{n}\right\}_{n=1}^{\infty}$ where $y_{n}=1-\frac{1}{n}$ for all $n \in \mathrm{N}$ in $[0,1]$. Since $\lim _{n \rightarrow \infty} d_{E^{2}}\left(\left(y_{n}, 1\right),(1,1)\right)=0$ so by our recent assumption

$$
\lim _{n \rightarrow \infty} d_{E}\left(\left(y_{n}, 1\right),(1,1)\right)=0
$$

Then, there exists a sub-sequence $\left\{y_{n_{k}}\right\}_{k=1}^{\infty}$ such that $d\left(y_{n}, 1\right)<\frac{1}{2^{k}}$ for all $k \in \mathrm{N}$. This implies that $\sum_{k=1}^{\infty} d\left(y_{n_{k}}, 1\right)<\infty$. Now if we define a function $f$ on $[0,1]$ by: $f(x)=y_{n_{k}}$ if $\left(x=y_{n_{k}}, k=1,2 \ldots\right)$ and $f(x)=1$ if $\left(x \neq y_{n_{k}}, k=1,2 \ldots\right)$, then

$$
V_{d}(f,[0,1]) \leq 2\left(\sup _{0 \leq x, y \leq 1} d(x, y)+\sum_{k=1}^{\infty} d\left(y_{n_{k}}, 1\right)\right)<\infty
$$

Furthermore, we consider $g$ on $[0,1]$ defined by: $g(x)=0$ if $(x=1)$ and $g(x)=1$ if $(x \neq$ 1). Then $V_{d}(g,[0,1])=d(0,1)<\infty$. Finally $g \circ f$ is defined on $[0,1]$ by: $g \circ f(x)=1$ if $\left(x=y_{n_{k}}, k=1,2 \ldots\right)$, and $g \circ f(x)=0$ if $\left(x \neq y_{n_{k}}, k=1,2 \ldots\right)$, which implies $V_{d}(g \circ f,[0,1])=\infty$. Thus, by Definition 2 we have a contradiction to the assumption that the metric $d$ has the IBVCO property.

As the first remark, we point out that Theorem 3 gives merely a necessary condition on having the IBVCO property. For example, define a metric

$$
\begin{aligned}
& d:\left(\Re^{2}, d_{E^{2}}\right) \rightarrow\left(\Re, d_{E}\right) \\
& d(x, y)=d_{E}(h(x), h(y))
\end{aligned}
$$

Where the bijection $h: \mathfrak{R} \rightarrow \mathfrak{R}$ is defined by: $h(x)=x$ if $x \in(0,1), h(x)=-x+1$, and $h(x)=-x+1$ if $x \notin(0,1)$. The metric $d$ is discontinuous. To prove it consider the sequence $\quad\left\{\left(\frac{1}{n}, 0\right)\right\}_{n=1}^{\infty} \quad$ where $\lim _{n \rightarrow \infty} d_{E^{2}}\left(\left(\frac{1}{n}, 0\right),(0,0)\right)=1 \neq 0$. Now, define functions $f$ and $g$ similar to Example $2 . \quad$ In this case $\quad V_{d}(f,[0,1])=2 \sum_{k=1}^{\infty} \frac{1}{k^{2}}<\infty \quad$ and $V_{d}(g,[0,1])=2<\infty$. For the composite function $g \circ f$, taking the partitions $P_{n}$ as were taken in Example 2 gives: $V_{d}\left(g \circ f, P_{n}\right)=2 \sum_{k=1}^{n-1} \frac{1}{k}$ where $n=2,3 \ldots$ Accordingly, $V_{d}(g \circ f,[0,1])=\infty$.

To find the appropriate metrics $d$ that have the IBVCO property, Theorem 3 suggests that we should focus our attention on all discontinuous metrics $d:\left(\mathfrak{R}^{2}, d_{E^{2}}\right) \rightarrow\left(\mathfrak{R}, d_{E}\right)$. The next theorem discusses the above mentioned point.

Theorem 4. Let the metric $d$ satisfy the condition:

$$
\inf _{-\infty<x \neq y<\infty} d(x, y)>0 .
$$


Then, it has the IBVCO property.

Proof. First, let $f$ be an arbitrary function of bounded $d$ variation on $[a, b]$. We claim that $f$ is a step function. Suppose that this is not the case, so that there exists an increasing sequence $\left\{x_{n}\right\}_{n=1}^{\infty}$ of $[a, b]$ such that $f\left(x_{n}\right) \neq f\left(x_{n+1}\right)$ for all $n \in \mathrm{N}$. Hence, taking the partitions $P_{n}=$ $\left\{a, x_{1}, x_{2}, \ldots, x_{n}, b\right\}$ of $[a, b]$ gives:

$$
\begin{aligned}
V_{d}\left(f, P_{n}\right) & =d\left(f(a), f\left(x_{1}\right)\right)+\sum_{k=2}^{n} d\left(f\left(x_{k}\right), f\left(x_{k-1}\right)\right)+d\left(f\left(x_{n}\right), f(b)\right) \\
& \geq(n-1) \inf _{-\infty<x \neq y<\infty} d(x, y) \quad \text { for } n=1,2 \ldots
\end{aligned}
$$

So by definition, $V_{d}(f,[a, b])=\infty$ which is a contradiction to our assumption. Second, since $f$ is a step function, so for any function of bounded $d$ variation $g$ on $[c, d]$ with $\operatorname{Im}(f) \subseteq[c, d], g \circ f$ is also a step function on $[a, b]$. eventually, every step function is of bounded $d$ variation on $[a, b]$ and this completes the proof.

Corollary 5. The discrete metric $d_{0}$ has the IBVCO property.

As the second remark, we point to the cardinality of the set of all metrics $d:\left(\mathfrak{R}^{2}, d_{E^{2}}\right) \rightarrow\left(\mathfrak{R}, d_{E}\right)$ that have the IBVCO property is equal to the cardinality of the set of all metrics, that is, $2^{c}$ where $c$ denotes the continuum. To Prove this, for any function $f: \mathfrak{R} \rightarrow \mathfrak{R}$ with $\inf _{-\infty<x<\infty} f(x)>0$, define a metric $d_{f}$ by:

$$
\begin{aligned}
& d_{f}:\left(\Re^{2}, d_{E^{2}}\right) \rightarrow\left(\Re, d_{E}\right) \\
& d_{f}(x, y)=(f(x)+f(y)) d_{0}(x, y) .
\end{aligned}
$$

Now, since the cardinality of the set of all functions $\quad f: \mathfrak{R} \rightarrow \mathfrak{R} \quad$ with $\inf _{-\infty<x<\infty} f(x)>0$ is $2^{c}$ and for each element of this set $\inf _{-\infty<x \neq y<\infty} d_{f}(x, y) \geq 2 \inf _{-\infty<x<\infty} f(x)$, by means of Theorem 4 the claimed assertion is proved.

In the end, while Theorem 3 gives a necessary condition and theorem 4 gives a sufficient condition for the metric $d$ to have the IBVCO property, it remains for the interested reader to answer the following:

Question 2. Is there any necessary and sufficient condition of the metric $d$ to have the IBVCO property? If so, what is that condition?

\section{ACKNOWLEDGEMENTS}

The author would like to thanks Dr. F. Malek and Dr. K. Nourouzi for their time and advice. Special thanks to Dr. H. Haghighi for his invaluable support.

\section{REFERENCES}

1. H.S. Gaskill and P.P. Narayanaswami, Foundations of Analysis (Harper \& Row Publishers, New York, 1989), pages 320-326.

2. M.J. Schramm, Introduction to Real Analysis (Prentice-Hall, Englewood Cliffs, NJ, 1996), pages 300-302.

3. J.D. Depree and C.W. Swartz, Introduction to Real Analysis (Wiley, New York, 1988), pages 164-171.

4. W. Rudin, Principles of Mathematical Analysis (McGraw Hill, New York, 1988), pages 85, 105, 127.

5. V.V. Chistyako, "On Mapping of Bounded Variation," J. Dynam. Control Systems, Vol. 3, No. 2 (1997), pages 261-289.

6. W.A. Sutherland, Introduction to Metric and Topological Spaces (Clarendon press, Oxford, 1975).

7. C.C. Pugh, Real Mathematical Analysis (Springer-Verlag, NewYork, 2002). 


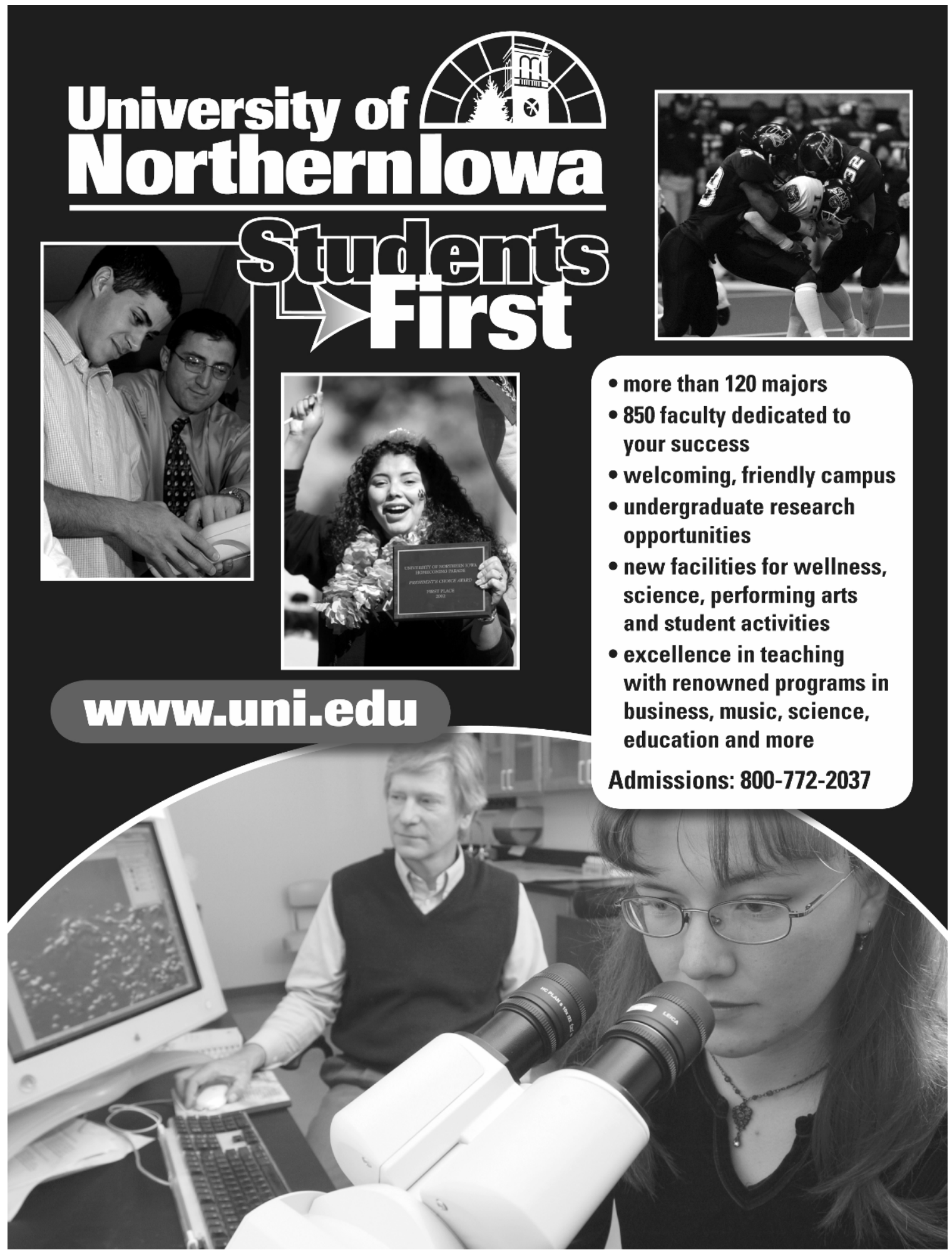

